\title{
ON IRREDUCIBLE MODULES OVER $q$-SKEW POLYNOMIAL RINGS AND SMASH PRODUCTS
}

\author{
PIOTR GRZESZCZUK
}

(Communicated by Birge Huisgen-Zimmermann)

\begin{abstract}
Let $M$ be an irreducible left module over a $q$-skew polynomial ring $R[x ; \sigma, \delta]$. We give sufficient conditions for the complete reducibility of $M$ considered as a module over the coefficient ring $R$. We apply it to irreducible modules over smash product $R \# H$, where $H$ is a Hopf algebra generated by skew primitive elements.
\end{abstract}

\section{INTRODUCTION}

For a given extension $R \subseteq S$ of associative rings (with the same unity), it is natural to ask whether (or when) irreducible left $S$-modules are completely reducible as $R$-modules. This question has a positive answer for several classes of "finite type" extensions: for example,

(i) finite normalizing extensions $R \subseteq \sum_{i=1}^{n} R s_{i}$ ([2]),

(ii) fixed rings of a finite group actions $R^{G} \subseteq R$, with $|G|^{-1} \in R([8]$ ),

(iii) rings graded by finite groups $R_{1} \subseteq \bigoplus_{g \in G} R_{g}$ ([4]).

In this paper we study some extensions of "infinite type"; namely, we consider modules over $q$-skew polynomial rings. We show that, under certain conditions, for a given left $R[x ; \sigma, \delta]$-module $M$ its socle $\operatorname{Soc}\left({ }_{R} M\right)$ over $R$ is also a module over the ring $R[x ; \sigma, \delta]$. Our conditions imply in particular that if $q$ is not a root of 1 , then:

1. finite dimensional irreducible $R[x ; \sigma, \delta]$-modules are completely reducible over $R$;

2. if $R$ is left socular (e.g., left artinian or right perfect), then irreducible left $R[x ; \sigma, \delta]$-modules are completely reducible over $R$.

As a consequence of our results on modules over $q$-skew polynomial rings, we obtain a description of certain modules over smash products $R \# H$, where $H$ is a Hopf algebra generated by skew primitive elements. Namely, we show that if $H$ is a character Hopf algebra (see [5]) over the field $k$ of characteristic 0 , and $\chi^{h}(g)$ is not an $n^{\text {th }}$ primitive root of $1(n>1)$ for any character skew $g$-primitive element $h \in H$, then

3. every finite dimensional irreducible left $R \# H$-module is completely reducible as a left $R$-module;

Received by the editors November 19, 2011 and, in revised form, February 26, 2012.

2010 Mathematics Subject Classification. Primary 16N20, 16S36, 16W25, 16S40.

Key words and phrases. Irreducible module, skew polynomial ring, skew derivation.

The author was supported in part by the grant MNiSW nr N N201 268435 and by the grant S/WI/1/2011 of Białystok University of Technology. 
4. if $R$ is left socular, then irreducible left $R \# H$-modules are completely reducible as left $R$-modules. Thus $\mathcal{J}(R) \subseteq \mathcal{J}(R \# H)$, where $\mathcal{J}$ is the Jacobson radical.

On the other hand, we should also point out that in the case where $H$ is finite dimensional and pointed, there is a strong relationship between the Jacobson radicals of $R$ and the crossed product $R \# H$; namely, it is proved in [7] that $\mathcal{J}(R \# H)^{\operatorname{dim}_{k} H} \subseteq \mathcal{J}(R) \cdot(R \# H)$.

We will now introduce the terminology and notation that will be used throughout the paper. Let $R$ be an associative ring and $\sigma$ be an automorphism of $R$. Then the additive map $\delta: R \rightarrow R$ is a $\sigma$-derivation if

$$
\delta(a b)=\delta(a) b+\sigma(a) \delta(b)
$$

for all $a, b \in R$. Suppose that $q$ is a nonzero central $(\sigma, \delta)$-constant in $R$, i.e., $\sigma(q)=q$ and $\delta(q)=0$. If $\delta \sigma=q \sigma \delta$, then $\delta$ is called a $q$-skew $\sigma$-derivation. If in addition $R$ is a $k$-algebra, we assume that $q \in k^{\times}$. The following $q$-Leibniz Rules hold in $R$ and $R[x ; \sigma, \delta]$ :

$$
\delta(a b)=\sum_{i=0}^{n}\left(\begin{array}{c}
n \\
i
\end{array}\right)_{q} \sigma^{n-i} \delta^{i}(a) \delta^{n-i}(b) \text { and } x^{n} a=\sum_{i=1}^{n}\left(\begin{array}{c}
n \\
i
\end{array}\right)_{q} \sigma^{n-i} \delta^{i}(a) x^{n-i}
$$

for all $a, b \in R$ and $n \geqslant 0$. The Gaussian $q$-binomial coefficient $\left(\begin{array}{c}n \\ i\end{array}\right)_{q}$ is defined as the evaluation at $t=q$ of the polynomial function

$$
\left(\begin{array}{c}
n \\
i
\end{array}\right)_{t}=\frac{\left(t^{n}-1\right)\left(t^{n-1}-1\right) \ldots\left(t^{n-i+1}-1\right)}{\left(t^{i}-1\right)\left(t^{i-1}-1\right) \ldots(t-1)}
$$

We will use the following $q$-Pascal identity:

$$
\left(\begin{array}{c}
n \\
i
\end{array}\right)_{q}=\left(\begin{array}{c}
n-1 \\
i
\end{array}\right)_{q}+q^{n-i}\left(\begin{array}{c}
n-1 \\
i-1
\end{array}\right)_{q}=\left(\begin{array}{c}
n-1 \\
i-1
\end{array}\right)_{q}+q^{i}\left(\begin{array}{c}
n-1 \\
i
\end{array}\right)_{q}
$$

for $n>i>0$ (cf. [3]).

We will say that the ring $R$ has $q$-characteristic zero if $1+q+\cdots+q^{m}$ is invertible in $R$, for any integer $m \geqslant 1$. If in addition $R$ is a $k$-algebra, then either $q$ is not a root of unity or $q=1$ and $\operatorname{char} k=0$.

If $r \in R$, then a left $R$-module $M$ is said to be $r$-torsion free if $r m \neq 0$ for all nonzero $m \in M$. If for any $m \in M$ there exists an integer $n=n(m)$ such that $r^{n} m=0$, then $M$ is called an $r$-torsion module.

A submodule $E$ of an $R$-module $M$ is said to be essential if $E \cap X \neq 0$ for any nonzero submodule $X \subseteq M$. It is well known that the intersection of all essential submodules of an $R$-module $M$ is equal to the sum of all irreducible submodules of $M$ and is called the socle of $M$, denoted by $\operatorname{Soc}(M)$. Finally, $\operatorname{Sing}(M)$ will be the singular submodule of $M$; that is, $\operatorname{Sing}(M)=\{m \in M$ | $\operatorname{ann}_{R}(m)$ is essential in ${ }_{R} R$ \}.

\section{2. $\boldsymbol{m}$-SEQUENCES AND ESSENTIAL SUBMODUlES}

Let $R[x ; \sigma, \delta]$ be a $q$-skew polynomial ring and $M$ a left $R[x ; \sigma, \delta]$-module. Let $E$ be an essential $R$-submodule of $M$ and $0 \neq m \in E$. By an $m$-sequence we mean a sequence $\mathbf{r}=\left\{r_{n}\right\}_{n \geqslant 0}$ of elements of $R$ satisfying the following properties:

$1^{\circ} \quad \sigma^{n}\left(r_{n}\right) x^{n} m \in E$ for all $n \geqslant 0$ and $\sigma^{s}\left(r_{s}\right) x^{s} m \neq 0$ for some $s$; 
$2^{\circ}$ if $\sigma^{n+1}\left(r_{n}\right) x^{n+1} m \in E$, then $r_{n+1}=r_{n}$;

$3^{\circ}$ if $\sigma^{n+1}\left(r_{n}\right) x^{n+1} m \notin E$, then $r_{n+1} \in R r_{n}$ and $\sigma^{n+1}\left(r_{n+1}\right) x^{n+1} m \in E \backslash\{0\}$.

The smallest integer $s$ such that $\sigma^{s}\left(r_{s}\right) x^{s} m \neq 0$ we denote by $\operatorname{deg} \mathbf{r}$ and call the degree of $\mathbf{r}$.

Lemma 1. If $a \in R$ and $\sigma^{s}(a) x^{s} m \neq 0$ for some $s \geqslant 1$, then there exists an $m$-sequence $\mathbf{r}=\left\{r_{n}\right\}_{n \geqslant 0}$ such that $r_{0}=a$ and $\operatorname{deg} \mathbf{r} \leqslant s$.

Proof. The sequence $\mathbf{r}$ we define inductively starting with $r_{0}=\cdots=r_{i-1}=a$, where $i$ is the smallest integer such that $\sigma^{i}(a) x^{i} m \notin E$. If such an $i$ does not exist, the constant sequence $\mathbf{r}=\{a\}$ satisfies the desired property. Next suppose that $j \geqslant i-1$ and $r_{0}, \ldots, r_{j}$ are given. If $\sigma^{j+1}\left(r_{j}\right) x^{j+1} m \in E$, then we put $r_{j+1}=r_{j}$. If $\sigma^{j+1}\left(r_{j}\right) x^{j+1} m \notin E$, then by essentiality of $E$ there exists $0 \neq c=\sigma^{j+1}\left(r^{\prime}\right) \in R$ such that

$$
0 \neq c \sigma^{j+1}\left(r_{j}\right) x^{j+1} m=\sigma^{j+1}\left(r^{\prime} r_{j}\right) x^{j+1} m \in E .
$$

In this situation we put $r_{j+1}=r^{\prime} r_{j}$. Clearly the sequence $\mathbf{r}$ satisfies conditions $1^{\circ}-3^{\circ}$, and from the construction it follows immediately that $\operatorname{deg} \mathbf{r} \leqslant s$.

An $m$-sequence $\mathbf{r}=\left\{r_{n}\right\}_{n \geqslant 0}$ is said to be weak if $r_{j}=r_{j+1}$ for some $j \geqslant \operatorname{deg} \mathbf{r}$. If $r_{j} \neq r_{j+1}$ for all $j \geqslant \operatorname{deg} \mathbf{r}$, we call $\mathbf{r}$ a strict $m$-sequence. Note that if $\mathbf{r}$ is strict and $j \geqslant \operatorname{deg} \mathbf{r}$, then $\sigma^{j}\left(r_{j}\right) x^{j} m \neq 0$. Indeed, if $\sigma^{j}\left(r_{j}\right) x^{j} m=0$, then $\sigma^{j}\left(r_{j-1}\right) x^{j} m$ must equal 0 , and hence $r_{j}=r_{j-1}$.

Lemma 2. Suppose that every $m$-sequence in $R$ is strict. Then:

(1) if $a \in R$ is such that $0 \neq a x^{l} m \in E$, then $\sigma(a) x^{l+1} m \notin E$;

(2) if $\mathbf{r}=\left\{r_{n}\right\}_{n \geqslant 0}$ is an $m$-sequence and $l \geqslant \operatorname{deg} \mathbf{r}$, then $\sigma^{j}\left(r_{l}\right) x^{j} m=0$ for all $j<l$;

(3) $\operatorname{ann}\left(x^{j+1} m\right) \subseteq \sigma^{-1}\left(\operatorname{ann}\left(x^{j} m\right)\right)$ for all $j \geqslant 0$.

Proof. 1. Suppose that $0 \neq a x^{l} m \in E$ and $\sigma(a) x^{l+1} m \in E$. By Lemma 1 we can take an $m$-sequence $\mathbf{r}$ such that $r_{0}=\sigma^{-l}(a)$ and $\operatorname{deg} \mathbf{r} \leqslant l$. Then $r_{l}=b r_{0}=b \sigma^{-l}(a)$, where $b \in R$. Notice that

$$
\sigma^{l+1}\left(r_{l}\right) x^{l+1} m=\sigma^{l+1}(b) \sigma(a) x^{l+1} m \in E .
$$

Hence $r_{l}=r_{l+1}$, contradicting our assumption that every $m$-sequence in $R$ is strict.

2. Suppose that $\sigma^{j}\left(r_{l}\right) x^{j} m \neq 0$ for some $j<l$. From the definition of an $m$-sequence it follows that we can choose $a, b \in R$ such that $r_{l}=a r_{j}=b r_{j+1}$. Then $0 \neq \sigma^{j}\left(r_{l}\right) x^{j} m=\sigma^{j}(a) \sigma^{j}\left(r_{j}\right) x^{j} m \in E$. On the other hand, $\sigma^{j+1}\left(r_{l}\right) x^{j+1} m=$ $\sigma^{j+1}(b) \sigma^{j+1}\left(r_{j+1}\right) x^{j+1} m \in E$, which is impossible by 1 .

3. Suppose $a \in R$ is such that $\sigma(a) x^{j+1} m=0$. By item 1, it follows that either $a x^{j} m=0$ or $a x^{j} m \notin E$. If $a x^{j} m \notin E$, then there exists $r \in R$ such $0 \neq \operatorname{rax}^{j} m \in E$. But in this situation $0=\sigma(r a) x^{j+1} m \in E$. By item 1 we obtain that $a x^{j} m$ must be equal to 0 ; thus $\operatorname{ann}\left(x^{j+1} m\right) \subseteq \sigma^{-1}\left(\operatorname{ann}\left(x^{j} m\right)\right)$.

Corollary 3. If every $m$-sequence in $R$ is strict, then $R$ contains an infinite strictly descending chain of left ideals

$$
\operatorname{ann}(m) \nsupseteq \sigma^{-1}(\operatorname{ann}(x m)) \supseteq \cdots \nsupseteq \sigma^{-l}\left(\operatorname{ann}\left(x^{l} m\right)\right) \supseteq \cdots \cdots
$$

Proof. Lemma 2(3) implies that $\sigma^{-l}\left(\operatorname{ann}\left(x^{l} m\right)\right) \subseteq \sigma^{-(l-1)}\left(\operatorname{ann}\left(x^{l-1} m\right)\right)$ for any $l>0$. To see that the inclusion is strict, it is enough to consider an $m$-sequence $\mathbf{r}$ of degree $\leqslant l-1$. Then Lemma $2(2)$ yields that $r_{l} \in \sigma^{-(l-1)}\left(\operatorname{ann}\left(x^{l-1} m\right)\right)$, but clearly $r_{l} \notin \sigma^{-l}\left(\operatorname{ann}\left(x^{l} m\right)\right)$. 
Lemma 4. If $R$ contains a weak $m$-sequence, then there exists an element $r \in R$ and a nonnegative integer $n$ such that

1. $0 \neq \sigma^{n}(r) x^{n} m \in E$ and $\sigma^{n+1}(r) x^{n+1} m \in E$,

2. $r m=\sigma(r) x m=\cdots=\sigma^{n-1}(r) x^{n-1}=0$.

Proof. Let $l \geqslant \operatorname{deg}(\mathbf{r})$ be the smallest integer with respect to the equality $r_{l}=r_{l+1}$. Then $\sigma^{l}\left(r_{l}\right) x^{l} m \neq 0$. Otherwise, if $\sigma^{l}\left(r_{l}\right) x^{l} m=0$, then from the definition it follows that $\sigma^{l}\left(r_{l-1}\right) x^{l} m \in E$, and hence $r_{l-1}=r_{l}$. Next consider the smallest integer $n$ with respect to $\sigma^{n}\left(r_{l}\right) x^{n} m \neq 0$. It is clear that $n \leqslant l$. Note that if $j \leqslant l$, then $r_{l}=s_{j} r_{j}$ for some $s_{j} \in R$. Thus $\sigma^{j}\left(r_{l}\right) x^{j} m=\sigma^{j}\left(s_{j}\right) \sigma^{j}\left(r_{j}\right) x^{j} m \in E$. Therefore $r=r_{l}$ and $n$ satisfy the lemma.

Lemma 5. Let $M$ be a q-torsion free left $R[x ; \sigma, \delta]$-module and $r \in R, m \in M$ be such that

$$
r m=\sigma(r) x m=\cdots=\sigma^{n-1}(r) x^{n-1} m=0 .
$$

Then $\sigma^{i} \delta^{j}(r) x^{i} m=0$ if $i+j \leqslant n-1$, and $\sigma^{n}(r) x^{n} m=(-1)^{n} q^{\frac{n(n-1)}{2}} \delta^{n}(r) m$.

Proof. First we show that if $i, j$ are nonnegative integers and $i+j \leqslant n-1$, then $\sigma^{i} \delta^{j}(r) x^{i} m=0$.

Suppose that $\sigma^{i} \delta^{j}(r) x^{i} m \neq 0$ and take $i, j$ such that the sum $i+j$ is possibly minimal. Next take $j$ possibly minimal. By assumption it follows that $j>0$, so

$$
\sigma^{i+1} \delta^{j-1}(r) x^{i+1} m=0 \text { and } \sigma^{i} \delta^{j-1}(r) x^{i} m=0 .
$$

Thus

$$
\begin{aligned}
0=x\left(\sigma^{i} \delta^{j-1}(r) x^{i} m\right) & =\sigma^{i+1} \delta^{j-1}(r) x^{i+1} m+\delta \sigma^{i} \delta^{j-1}(r) x^{i} m \\
& =q^{i} \sigma^{i} \delta^{j}(r) x^{i} m,
\end{aligned}
$$

a contradiction. The above implies, in particular, that if $i+j=n-1$, then

$$
0=x\left(\sigma^{i} \delta^{j}(r) x^{i} m\right)=\sigma^{i+1} \delta^{j}(r) x^{i+1} m+q^{i} \sigma^{i} \delta^{j+1}(r) x^{i} m .
$$

Hence

$$
\begin{gathered}
\sigma^{n}(r) x^{n} m=-q^{n-1} \sigma^{n-1} \delta(r) x^{n-1} m=q^{n-1} q^{n-2} \sigma^{n-2} \delta^{2}(r) x^{n-2} m \\
=\cdots=(-1)^{n} q^{n-1} q^{n-2} \ldots q \delta^{n}(r) m=(-1)^{n} q^{\frac{n(n-1)}{2}} \delta^{n}(r) m .
\end{gathered}
$$

For $1 \leqslant i, j \leqslant n$ let $a_{i j}=\left(\begin{array}{c}i+1 \\ j\end{array}\right)_{q} q^{(n-i) j}$, where $\left(\begin{array}{c}i+1 \\ j\end{array}\right)_{q}$ denotes the Gaussian $q$-binomial coefficient (see (1) in the Introduction). Let

$$
D_{n}=\operatorname{det}\left[a_{i j}\right]=\operatorname{det}\left[\begin{array}{lllll}
\left(\begin{array}{l}
2 \\
1
\end{array}\right)_{q} q^{n-1} & \left(\begin{array}{l}
2 \\
2
\end{array}\right)_{q} q^{2(n-1)} & 0 & \ldots & 0 \\
\left(\begin{array}{l}
3 \\
1
\end{array}\right)_{q} q^{n-2} & \left(\begin{array}{l}
3 \\
2
\end{array}\right)_{q} q^{2(n-2)} & \left(\begin{array}{l}
3 \\
3
\end{array}\right)_{q} q^{3(n-2)} & \ldots & 0 \\
\ldots & \ldots & \ldots & \ldots & \ldots \\
\left(\begin{array}{c}
n \\
1
\end{array}\right)_{q} q & \left(\begin{array}{c}
n \\
2
\end{array}\right)_{q} q^{2} & \left(\begin{array}{c}
n \\
3
\end{array}\right)_{q} q^{3} & \ldots & \left(\begin{array}{c}
n \\
n
\end{array}\right)_{q} q^{n} \\
\left(\begin{array}{c}
n+1 \\
1
\end{array}\right)_{q} & \left(\begin{array}{c}
n+1 \\
2
\end{array}\right)_{q} & \left(\begin{array}{c}
n+1 \\
3
\end{array}\right)_{q} & \ldots & \left(\begin{array}{c}
n+1 \\
n
\end{array}\right)_{q}
\end{array}\right] .
$$

Lemma 6. $D_{n}=q^{\frac{n^{3}-n}{6}}\left(1+q+\cdots+q^{n}\right)$. 
Proof. Notice that by using the $q$-Pascal identity,

$$
\begin{gathered}
a_{i+1, j}=\left(\begin{array}{c}
i+2 \\
j
\end{array}\right)_{q} q^{(n-i-1) j}=\left(\begin{array}{c}
i+1 \\
j-1
\end{array}\right)_{q} q^{(n-i-1) j}+\left(\begin{array}{c}
i+1 \\
j
\end{array}\right)_{q} q^{j} q^{(n-i-1) j} \\
=\left(\begin{array}{c}
i+1 \\
j-1
\end{array}\right)_{q} q^{(n-i-1) j}+a_{i j} .
\end{gathered}
$$

The above implies that

$$
\begin{aligned}
D_{n}=\operatorname{det} & {\left[\begin{array}{lllll}
\left(\begin{array}{l}
2 \\
1
\end{array}\right)_{q} q^{n-1} & \left(\begin{array}{l}
2 \\
2
\end{array}\right)_{q} q^{2(n-1)} & 0 & \ldots & 0 \\
\left(\begin{array}{l}
2 \\
0
\end{array}\right)_{q} q^{n-2} & \left(\begin{array}{l}
2 \\
1
\end{array}\right)_{q} q^{2(n-2)} & \left(\begin{array}{l}
2 \\
2
\end{array}\right)_{q} q^{3(n-2)} & \ldots & 0 \\
\ldots & \ldots & \ldots & \ldots & \ldots \\
\left(\begin{array}{c}
n-1 \\
0
\end{array}\right)_{q} q & \left(\begin{array}{c}
n-1 \\
1
\end{array}\right)_{q} q^{2} & \left(\begin{array}{c}
n-1 \\
2
\end{array}\right)_{q} q^{3} & \ldots & \left(\begin{array}{c}
n-1 \\
n-1
\end{array}\right)_{q} q^{n} \\
\left(\begin{array}{l}
n \\
0
\end{array}\right)_{q} & \left(\begin{array}{l}
n \\
1
\end{array}\right)_{q} & \left(\begin{array}{l}
n \\
2
\end{array}\right)_{q} & \ldots & \left(\begin{array}{c}
n \\
n-1
\end{array}\right)_{q}
\end{array}\right] } \\
= & \left(\begin{array}{l}
2 \\
1
\end{array}\right)_{q} q^{n-1} q^{n-2} \ldots q \cdot D_{n-1}-\left(\begin{array}{l}
2 \\
2
\end{array}\right)_{q} q^{2(n-1)} W_{n-1},
\end{aligned}
$$

where

$$
W_{n-1}=\operatorname{det}\left[\begin{array}{lllll}
q^{n-2} & \left(\begin{array}{l}
2 \\
2
\end{array}\right)_{q} q^{3(n-2)} & 0 & \ldots & 0 \\
q^{n-3} & \left(\begin{array}{l}
3 \\
2
\end{array}\right)_{q} q^{3(n-3)} & \left(\begin{array}{l}
3 \\
3
\end{array}\right)_{q} q^{4(n-3)} & \ldots & 0 \\
\ldots & \ldots & \ldots & \ldots & \ldots \\
q & \left(\begin{array}{c}
n-1 \\
2
\end{array}\right)_{q} q^{3} & \left(\begin{array}{c}
n-1 \\
3
\end{array}\right)_{q} q^{4} & \ldots & \left(\begin{array}{c}
n-1 \\
n-1
\end{array}\right)_{q} q^{n} \\
1 & \left(\begin{array}{c}
n \\
2
\end{array}\right)_{q} & \left(\begin{array}{c}
n \\
3
\end{array}\right)_{q} & \ldots & \left(\begin{array}{c}
n \\
n-1
\end{array}\right)_{q}
\end{array}\right]
$$

Again applying the $q$-Pascal identity, one immediately obtains that

$$
\begin{gathered}
W_{n-1}=\operatorname{det}\left[\begin{array}{lllll}
q^{n-2} & \left(\begin{array}{l}
2 \\
2
\end{array}\right)_{q} q^{3(n-2)} & 0 & \ldots & 0 \\
0 & \left(\begin{array}{l}
2 \\
1
\end{array}\right)_{q} q^{3(n-3)} & \left(\begin{array}{l}
2 \\
2
\end{array}\right)_{q} q^{4(n-3)} & \ldots & 0 \\
\ldots & \ldots & \ldots & \ldots & \ldots \\
0 & \left(\begin{array}{c}
n-2 \\
1
\end{array}\right)_{q} q^{3} & \left(\begin{array}{c}
n-2 \\
2
\end{array}\right)_{q} q^{4} & \ldots & \left(\begin{array}{c}
n-2 \\
n-2
\end{array}\right)_{q} q^{n} \\
0 & \left(\begin{array}{c}
n-1 \\
1
\end{array}\right)_{q} & \left(\begin{array}{c}
n-1 \\
2
\end{array}\right)_{q} & \ldots & \left(\begin{array}{c}
n-1 \\
n-2
\end{array}\right)_{q}
\end{array}\right] \\
=q^{n-2} q^{2(n-3)} q^{2(n-4)} \ldots q^{2} \cdot D_{n-2}=q^{n^{2}-4 n+4} D_{n-2} .
\end{gathered}
$$

Thus

$$
D_{n}=(1+q) q^{\frac{n(n-1)}{2}} D_{n-1}-q^{n^{2}-2 n+2} D_{n-2}
$$

with $D_{1}=1+q$ and $D_{2}=q\left(1+q+q^{2}\right)$. The lemma now follows by an easy induction.

Proposition 7. Let $M$ be a left $R[x ; \sigma, \delta]$-module which is $D_{n}$-torsion free for all $n \geqslant 1$. Let $E$ be an essential $R$-submodule of $M$ such that for every $m \in E$, the ring $R$ contains a weak m-sequence. Then

$$
E \cap x^{-1} E=\{m \in E \mid x m \in E\}
$$

is also essential as an $R$-submodule. 
Proof. Notice that if $e \in E$ and $x e \in E$, then for every $r \in R$,

$$
\text { xre }=\sigma(r) x e+\delta(r) e \in E .
$$

Thus $E \cap x^{-1} E$ is an $R$-submodule of $M$.

Suppose that $E \cap x^{-1} E$ is not essential. Then there exists a nonzero element $m \in E$ such that $\left(E \cap x^{-1} E\right) \cap R m=0$. Since $R$ contains a weak $m$-sequence, by Lemma 4 we can take $r \in R$ and $n \geqslant 0$ such that

$$
\begin{gathered}
r m=\sigma(r) x m=\cdots=\sigma^{n-1}(r) x^{n-1} m=0, \\
0 \neq \sigma^{n}(r) x^{n} m \in E \text { and } \sigma^{n+1}(r) x^{n+1} m \in E .
\end{gathered}
$$

For $1 \leqslant i, j \leqslant n$, let $a_{i j}=\left(\begin{array}{c}i+1 \\ j\end{array}\right)_{q} q^{(n-i) j}$ and $x_{j}=\sigma^{n+1-j} \delta^{j}(r) x^{n+1-j} m$. Applying the $q$-Leibniz rule for $i=1,2, \ldots, n-1$, we obtain

$$
\begin{gathered}
0=x^{i+1}\left(\sigma^{n-i}(r) x^{n-i} m\right)=\sum_{j=0}^{i+1}\left(\begin{array}{c}
i+1 \\
j
\end{array}\right)_{q} \sigma^{i+1-j} \delta^{j} \sigma^{n-i}(r) x^{n+1-j} m \\
=\sum_{j=0}^{i+1}\left(\begin{array}{c}
i+1 \\
j
\end{array}\right)_{q} q^{(n-i) j} \sigma^{n+1-j} \delta^{j}(r) x^{n+1-j} m \\
=\sigma^{n+1}(r) x^{n+1} m+\sum_{j=1}^{i+1} a_{i j} x_{j} .
\end{gathered}
$$

Thus $\sum_{j=1}^{i+1} a_{i j} x_{j}=-\sigma^{n+1}(r) x^{n+1} m \in E$. Moreover, for $i=n$ we have

$$
0=x^{n+1} r m=\sigma^{n+1}(r) x^{n+1} m+\sum_{j=1}^{n} a_{n j} x_{j}+\delta^{n+1}(r) m,
$$

so $\sum_{j=1}^{n} a_{n j} x_{j} \in E$. Now it is clear that for any $j=1,2, \ldots, n$ the element $D_{n} x_{j} \in E$, where $D_{n}$ is the determinant from Lemma 6. We note that $D_{n} x_{1}=D_{n} \sigma^{n} \delta(r) x^{n} m \in$ $E$, so

$$
\begin{gathered}
x\left(D_{n} \sigma^{n}(r) x^{n} m\right)=D_{n} \sigma^{n+1}(r) x^{n+1} m+D_{n} \delta \sigma^{n}(r) x^{n} m \\
=D_{n} \sigma^{n+1}(r) x^{n+1} m+D_{n} q^{n} \sigma^{n} \delta(r) x^{n} m \in E .
\end{gathered}
$$

On the other hand, by Lemma 5, $\sigma^{n}(r) x^{n} m=(-1)^{n} q^{\frac{n(n-1)}{2}} \delta^{n}(r) m$ and $M$ is $D_{n^{-}}$ torsion free; thus

$$
0 \neq D_{n} \sigma^{n}(r) x^{n} m \in\left(E \cap x^{-1} E\right) \cap R m,
$$

a contradiction. Therefore $E \cap x^{-1} E$ is an essential submodule of $M$.

Corollary 8. Let $M$ be a left $R[x ; \sigma, \delta]$-module which is $D_{n}$-torsion free for all $n \geqslant 1$. Suppose that for every essential $R$-submodule $E$ of $M$ and $0 \neq m \in E$, the ring $R$ contains a weak m-sequence. Then $\operatorname{Soc}\left({ }_{R} M\right)$ is an $R[x ; \sigma, \delta]$-module. In particular, if $M$ is simple as an $R[x ; \sigma, \delta]$-module, then either $\operatorname{Soc}\left({ }_{R} M\right)=0$ or ${ }_{R} M$ is completely reducible.

Proof. Let $m \in \operatorname{Soc}\left({ }_{R} M\right)$. If $E$ is an essential submodule of ${ }_{R} M$, then by Proposition $7 E \cap x^{-1} E$ is also essential, so $m \in E \cap x^{-1} E$. Hence $x m \in E$. Therefore $\operatorname{Soc}\left({ }_{R} M\right)$ is an $R[x ; \sigma, \delta]$-module. 


\section{Applications}

In this section we describe situations in which our condition on the existence of weak $m$-sequences is automatically satisfied.

Let $\Lambda$ be a well ordered set of ordinal numbers with the least element 0 . For a ring $R$ one can define a chain of ideals $\left\{S_{\alpha}\right\}_{\alpha \in \Lambda}$ as follows: $S_{0}=0$; if $\alpha \in \Lambda$, then $S_{\alpha+1} / S_{\alpha}=\operatorname{Soc}\left(R / S_{\alpha}\right)$, the left socle of $R / S_{\alpha}$. If $\beta \in \Lambda$ is a limit number, set $S_{\beta}=\bigcup_{\alpha<\beta} S_{\alpha}$. Recall that a $\operatorname{ring} R$ is said to be left socular (cf. [1]) if every nonzero left $R$-module contains a simple submodule. If $R$ is left socular, the set $\Lambda$ can be chosen such that $R=S_{\alpha}$ for some $\alpha \in \Lambda$. Note that the class of socular rings contains left artinian rings and right perfect rings.

If $A$ is a $k$-algebra, then $A$-module $M$ is locally finite dimensional if every finitely generated submodule of $M$ is finite dimensional.

Proposition 9. Let $M$ be a left $R[x ; \sigma, \delta]$-module and $E$ its essential $R$-submodule. Suppose that one of the following conditions is fulfilled:

1. $R$ is left socular;

2. $R$ is a left noetherian $k$-algebra and $M$ is locally finite dimensional as a $k[x]$-module;

3. $\operatorname{dim}_{k} M<\infty$;

4. there exists an integer $N$ such that $d^{N+1}(r) \in \sum_{j=0}^{N} R d^{j}(r)$ for all $r \in R$;

5. $M$ is x-torsion; i.e., for any $m \in M$ there exists $n=n(m)$ such that $x^{n} m=0$

6. $R$ is a k-algebra, $\sigma=\operatorname{id}_{R}$ and $M$ is locally finite dimensional as a $k[x]$ module.

Then for any nonzero $m \in E$ the ring $R$ contains a weak $m$-sequence.

Proof. 1. Suppose that $R$ is left socular. Let $\gamma$ be the smallest ordinal such that $S_{\gamma}$ contains an $m$-sequence $\left\{r_{l}\right\}_{l \geqslant 0}$. It is clear that $\gamma$ is not a limit ordinal. Note that if $a \in S_{\gamma-1}$, then $\sigma^{l}(a) x^{l} m=0$. Otherwise, we have an $m$-sequence $\left\{r_{l}^{\prime}\right\}_{l \geqslant 0}$ with $r_{0}^{\prime}=a \in S_{\gamma-1}$. Since $R r_{l}^{\prime} \supseteq R r_{l+1}^{\prime}$, one obtains that $r_{l}^{\prime} \in S_{\gamma-1}$ for all $l$. This contradicts minimality of $\gamma$.

Let $\varphi: R \rightarrow R / S_{\gamma-1}$ be the canonical homomorphism. Since $R r_{0} \supseteq R r_{1} \supseteq \cdots \supseteq$ $R r_{l} \supseteq \cdots$, we have a chain

$$
\varphi\left(R r_{0}\right) \supseteq \varphi\left(R r_{1}\right) \supseteq \cdots \supseteq \varphi\left(R r_{l}\right) \supseteq \cdots
$$

of cyclic submodules of a semisimple module $S_{\gamma} / S_{\gamma-1}$. Since $\varphi\left(R r_{0}\right)$ is contained in a finite direct sum of simple modules, this chain terminates. On the other hand, if $\varphi\left(R r_{l}\right)=\varphi\left(R r_{l+1}\right)$, then there exist $r^{\prime} \in R$ and $a \in S_{\gamma-1}$ such that $r_{l}=r^{\prime} r_{l+1}+a$. By the above, $\sigma^{l+1}(a) x^{l+1} m=0$, so

$$
\sigma^{l+1}\left(r_{l}\right) x^{l+1} m=\sigma^{l+1}\left(r^{\prime}\right) \sigma^{l+1}\left(r_{l+1}\right) x^{l+1} m \in E .
$$

From the definition of an $m$-sequence it follows that $r_{l}=r_{l+1}$. Therefore the sequence $\mathbf{r}$ is weak.

2. Suppose that every $m$-sequence in $R$ is strict. Corollary 3 tells us that the chain of left ideals

$$
\operatorname{ann}(m) \nsupseteq \sigma^{-1}(\operatorname{ann}(x m)) \supseteq \cdots \nsupseteq \sigma^{-l}\left(\operatorname{ann}\left(x^{l} m\right)\right) \supseteq \cdots
$$


is strict. Since $\operatorname{dim} \operatorname{span}_{F}\left(m, x m, x^{2} m, \ldots\right)<\infty$, there exists an integer $t$ such that $x^{n} m \in \operatorname{span}_{F}\left(m, x m, x^{2} m, \ldots, x^{t} m\right)$ for all $n \geqslant t$. Then

$$
\operatorname{ann}\left(m, x m, x^{2} m, \ldots, x^{t} m\right) \subseteq \operatorname{ann}\left(x^{n} m\right)
$$

for $n \geqslant t$, and consequently $\bigcap_{l=0}^{\infty} \operatorname{ann}\left(x^{l} m\right)=\bigcap_{l=0}^{t} \operatorname{ann}\left(x^{l} m\right)$. Set $I=\bigcap_{l=0}^{t} \operatorname{ann}\left(x^{l} m\right)$ and take $r \in I$. For any $l \geqslant 1, r \in \operatorname{ann}\left(x^{l} m\right)$, so

$$
\sigma^{-l}(r) \in \sigma^{-l}\left(\operatorname{ann}\left(x^{l} m\right)\right) \subseteq \sigma^{-(l-1)}\left(\operatorname{ann}\left(x^{l-1} m\right)\right) ;
$$

hence $\sigma^{-1}(r) \in \operatorname{ann}\left(x^{l-1} m\right)$. Then it follows that $\sigma^{-1}(I) \subseteq I$, and so $I \subseteq \sigma(I)$. The ring $R$ is left noetherian, so the chain $I \subseteq \sigma(I) \subseteq \sigma^{2}(I)$. . must stop. It implies immediately that $\sigma(I)=I$.

Next we claim that there exists an increasing sequence $\{f(n)\}_{n \geqslant 0}$ of nonnegative integers such that

$$
\sigma\left(\bigcap_{l=0}^{f(n)} \operatorname{ann}\left(x^{l} m\right)\right) \nsubseteq \bigcap_{j>f(n)} \operatorname{ann}\left(x^{j} m\right)
$$

We proceed by induction. By Corollary 3 we can put $f(0)=0$. Assume $n \geqslant 0$ $f(n)$

and let $a \in \bigcap_{l=0}^{n} \operatorname{ann}\left(x^{l} m\right)$ be such that $\sigma(a) x^{i} m \neq 0$ for some $i>f(n)$. Since $I$ is $\sigma$-stable, $a \notin I$, so there exists $s>f(n)$ such that $a \in \bigcap_{l=0}^{s-1} \operatorname{ann}\left(x^{l} m\right)$ and $a x^{s} m \neq 0$. Take $b \in R$ such that $0 \neq b a x^{s} m \in E$. If every $m$-sequence is strict, then by Lemma 2(1), $\sigma(b a) x^{s+1} m \notin E$. Since $E$ is essential, one can choose $c \in R$ such that $0 \neq \sigma(c b a) x^{s+1} m \in E$. Again by Lemma $2(1), c_{b a x}^{s} m=0$, so $c b a \in$ $\bigcap_{l=0}^{s} \operatorname{ann}\left(x^{l} m\right)$. Since $\sigma(c b a) x^{s+1} m \neq 0$, we have $\sigma\left(\bigcap_{l=0}^{s} \operatorname{ann}\left(x^{l} m\right)\right) \nsubseteq \bigcap_{j>s} \operatorname{ann}\left(x^{j} m\right)$. Thus it suffices to put $f(n+1)=s$. This proves the claim.

But now, if $f(n)>t$, then $I=\bigcap_{l=0}^{f(n)} \operatorname{ann}\left(x^{l} m\right)=\bigcap_{l=0}^{\infty} \operatorname{ann}\left(x^{l} m\right)$. Since $I$ is $\sigma$-stable,

$$
\sigma\left(\bigcap_{l=0}^{f(n)} \operatorname{ann}\left(x^{l} m\right)\right) \subseteq \bigcap_{l=0}^{\infty} \operatorname{ann}\left(x^{l} m\right) \subseteq \bigcap_{j>f(n)} \operatorname{ann}\left(x^{j} m\right),
$$

contradicting the definition of $f(n)$. Thus $R$ contains a weak $m$-sequence.

3. Let $P=\operatorname{ann}(M)$. Then $\operatorname{dim}_{F}(R / P)<\infty$ and $P \subseteq \operatorname{ann}\left(x^{l} m\right)$ for any $l$. Note that the mapping $a+\operatorname{ann}\left(x^{l} m\right) \longmapsto \sigma^{-l}(a)+\sigma^{-l}\left(\operatorname{ann}\left(x^{l} m\right)\right)$ provides an isomorphism of vector spaces $R / \operatorname{ann}\left(x^{l} m\right) \approx R / \sigma^{-l}\left(\operatorname{ann}\left(x^{l} m\right)\right)$. Thus

$$
\operatorname{dim}_{F} R / \sigma^{-l}\left(\operatorname{ann}\left(x^{l} m\right)\right) \leqslant \operatorname{dim}_{F}(R / P) .
$$

From Corollary 3 it follows that $R$ contains a weak $m$-sequence.

4. Let $\mathbf{r}=\left\{r_{n}\right\}_{n \geqslant 0}$ be a strict $m$-sequence with $\operatorname{deg} \mathbf{r} \leqslant N$. Then $\sigma^{j}\left(r_{N+1}\right) x^{j} m$ $=0$ for all $j \leqslant N$ and $\sigma^{N+1}\left(r_{N+1}\right) x^{N+1} m \neq 0$. By Lemma 5 ,

$$
0=\sigma^{j}\left(r_{N+1}\right) x^{j} m=(-1)^{j} q^{\frac{j(j-1)}{2}} \delta^{j}(r) m
$$


for all $j \leqslant N$. Thus

$$
\begin{aligned}
\sigma^{N+1}\left(r_{N+1}\right) x^{N+1} m & =(-1)^{N+1} \frac{N(N+1)}{2} \delta^{N+1}\left(r_{N+1}\right) m \\
& \in \sum_{j=0}^{N} R \delta^{j}\left(r_{N+1}\right) m=0,
\end{aligned}
$$

a contradiction. Consequently, in this situation, every $m$-sequence is weak.

5. This follows directly from Corollary 3 .

6. Suppose $\sigma=\mathrm{id}_{R}$. If every $m$-sequence in $R$ is strict, Corollary 3 says that the chain $\operatorname{ann}(m) \nsupseteq \operatorname{ann}(x m) \nsupseteq \cdots \nsupseteq \operatorname{ann}\left(x^{n} m\right) \nsupseteq \cdots$ is strict. But this contradicts our assumption that $\operatorname{span}_{F}\left\{m, x m, \ldots, x^{l} m \ldots\right\}$ is finite dimensional.

Recall that an automorphism $\sigma$ of the ring $R$ is said to be of locally finite order if for every $r \in R$, there exists an integer $n=n(r)>0$ such that $\sigma^{n}(r)=r$. If the ring $R$ is left socular, then nonzero left $R$-modules contain simple submodules. Therefore Proposition 9, condition 1, and Corollary 8 give us

Corollary 10. If $R$ is a left socular ring of q-characteristic zero, then simple left $R[x ; \sigma, \delta]$-modules are completely reducible as left $R$-modules. Thus the Jacobson radical $\mathcal{J}(R)$ is contained in the Jacobson radical $\mathcal{J}(R[x ; \sigma, \delta])$. Moreover, if the automorphism $\sigma$ has locally finite order, then

$$
\mathcal{J}(R[x ; \sigma, \delta])=\mathcal{J}(R)[x ; \sigma, \delta] .
$$

Proof. Since simple $R[x ; \sigma, \delta]$-modules are completely reducible as $R$-modules, we have $\mathcal{J}(R) \subseteq \mathcal{J}(R[x ; \sigma, \delta])$. Suppose that $\sigma$ has locally finite order. We know that $\mathcal{J}(R[x ; \sigma, \delta]) \cap R$ is a quasi-regular ideal of $R$, so $\mathcal{J}(R[x ; \sigma, \delta]) \cap R \subseteq \mathcal{J}(R)$ and consequently $\mathcal{J}(R[x ; \sigma, \delta]) \cap R=\mathcal{J}(R)$. This implies that $\mathcal{J}(R)$ is $\delta$-stable and

$$
R[x ; \sigma, \delta] / \mathcal{J}(R)[x ; \sigma, \delta] \simeq(R / \mathcal{J}(R))[x ; \widehat{\sigma}, \widehat{\delta}],
$$

where $\widehat{\sigma}$ is an induced automorphism and $\widehat{\delta}$ is a $q$-skew $\widehat{\sigma}$-derivation of $R / \mathcal{J}(R)$, respectively. Now it remains to prove that if $R$ is semiprimitive and socular, then $S=R[x ; \sigma, \delta]$ is semiprimitive. To this end, suppose that $\mathcal{J}(S) \neq 0$ and let $n$ be the minimum of degrees of nonzero polynomials from $\mathcal{J}(S)$. The set $\{0\} \cup\{a \mid$ $a x^{n}+g(x) \in \mathcal{J}(S)$, where $\left.\operatorname{deg} g(x)<n\right\}$ is a nonzero ideal of $R$. In particular, it contains a minimal left ideal of the form $I=R e$, where $e$ is a nonzero idempotent. Let $f(x)=e x^{n}+g(x) \in \mathcal{J}(S)$ and $m>0$ be such that $\sigma^{m}(e)=e$. By eventually replacing $f(x)$ by $f(x) x^{k}$, where $k$ is such that $\operatorname{deg} f(x) x^{k}$ is divisible by $m$, we have in the Jacobson radical of $S$ a nonzero polynomial $f(x)=e x^{l}+h(x)$ such that $e$ is a nonzero idempotent, $\sigma^{l}(e)=e$, and $\operatorname{deg} h(x)<l$. It is well known that $\mathcal{J}(e S e)=e \mathcal{J}(S) e$. Therefore

$$
e f(x) e=e x^{l} e+e h(x) e=e x^{l}+\widetilde{h}(x) \in \mathcal{J}(e S e),
$$

where $\widetilde{h}(x) \in e S e$. Let $e g(x) e \in e S e$ be a quasi-inverse for $e f(x) e$. Then $e g(x) e$ has a positive degree $s$ in $x$ and

$$
e f(x) e+e g(x) e=e f(x) e g(x) e .
$$

Since $e$ is the identity element in $e S e$, the right-hand side of the above equality has degree $n+s>\max \{n, s\} \geq \operatorname{deg}(e f(x) e+e g(x) e)$. Thus $\mathcal{J}(S)=0$. 
In [6] the authors considered the so-called "finite Jacobson radical" $\mathcal{J}_{\text {fin }}(R)$ of a $k$-algebra $R$, defined as the intersection of all the annihilators of all finite dimensional irreducible (left) $R$-modules. Thus by Proposition 9 , condition 3 , and Corollary 8 we have

Corollary 11. Let $R$ be a k-algebra with a q-skew $\sigma$-derivation $\delta$. If $R$ has $q$ characteristic zero, then every finite dimensional irreducible left $R[x ; \sigma, \delta]$-module is completely reducible as a left $R$-module. Thus

$$
\mathcal{J}_{\text {fin }}(R) \subseteq \mathcal{J}_{\text {fin }}(R[x ; \sigma, \delta]) .
$$

We note that $R$ can be viewed as a left $R[x ; \sigma, \delta]$-module with the action defined as

$$
\left(\sum_{i} a_{i} x^{i}\right) \cdot r=\sum_{i} a_{i} \delta^{i}(r)
$$

The $R[x ; \sigma, \delta]$-submodules of $R$ are precisely the left ideals of $R$ which are stable under $\delta$. Recall that $\delta$ is said to be locally algebraic if $R$ is locally finite dimensional as a left $k[x]$-module. Moreover in this case, if $m \in R$, then $\sigma^{-l}\left(\operatorname{ann}_{R}\left(x^{l} m\right)\right)=$ $\operatorname{ann}_{R}\left(\delta^{l}\left(\sigma^{-l}(m)\right)\right.$. Thus if $R$ satisfies descending chain condition on left annihilators, then Corollary 3 guarantees that for any essential left ideal $E$ and a nonzero element $m \in E$, the ring $R$ contains a weak $m$-sequence. Therefore we can apply Propositions 7, 9 and Corollary 8 to obtain the following.

Corollary 12. Let $R$ be a k-algebra of q-characteristic zero, with a q-skew $\sigma$ derivation $\delta$. Suppose that one of the following conditions is fulfilled:

(1) $R$ satisfies dcc on left annihilators;

(2) $R$ is left noetherian and $\delta$ is locally algebraic;

(3) $\delta$ is locally nilpotent;

(4) there exists an integer $N$ such that for any $r \in R, \delta^{N+1}(r) \in \sum_{j=0}^{N} R \delta^{j}(r)$;

(5) $\sigma=\operatorname{id}_{R}, q=1$ and the derivation $\delta$ is locally algebraic.

If $M$ is a left $R[x ; \sigma, \delta]$-module, then the singular submodule $\operatorname{Sing}\left({ }_{R} M\right)$ over $R$ is also an $R[x ; \sigma, \delta]$-submodule. The left socle $\operatorname{Soc}\left({ }_{R} R\right)$ of $R$ and left singular ideal $\operatorname{Sing}\left({ }_{R} R\right)$ are $\delta$-invariant. In addition, if $R$ contains a minimal left ideal and $R$ does not contain proper $\delta$-stable two-sided ideals, then $R$ is a semisimple artinian ring.

Proof. Let $m \in \operatorname{Sing}\left({ }_{R} M\right)$ and $L=\operatorname{ann}_{R}(m)$. If $L$ is an essential left ideal of $R$, then by Proposition [7 $\widehat{L}=L \cap \delta^{-1}(L)=\{r \in L \mid \delta(r) \in L\}$ is essential. It is also clear that $\sigma(\widehat{L})$ is essential, and for every $r \in \widehat{L}$,

$$
\sigma(r) x m=x r m-\delta(r) m=0 .
$$

Hence $\sigma(\widehat{L}) \subseteq \operatorname{ann}_{R}(x m)$ and $x m \in \operatorname{Sing}\left({ }_{R} M\right)$. Consequently, $\operatorname{Sing}\left({ }_{R} M\right)$ is an $R[x ; \sigma, \delta]$-submodule of $M$.

If $R$ contains a minimal ideal, then $\operatorname{Soc}\left({ }_{R} R\right)$ is a nonzero and $\delta$-stable ideal of $R$. Therefore if $R$ is $\delta$-simple, then $R=\operatorname{Soc}\left({ }_{R} R\right)$. Since $R$ has unity, $R$ is a finite direct sum of minimal left ideals.

Let $H$ be a Hopf algebra with comultiplication $\Delta$ and with the group $G$ of group-like elements, i.e., $G=\{g \in H \mid \Delta(g)=g \otimes g\}$. For $g \in G$, let

$$
L_{g}=\{h \in H \mid \Delta(h)=h \otimes 1+g \otimes h\}
$$


be the subspace of $g$-primitive (skew primitive) elements. It is clear that the group $G$ acts on $H$ by the conjugations $h^{g}=g^{-1} h g$ and that the subspace $L=\bigoplus_{g \in G} L_{g}$ is $G$-stable under this action. Following [5], recall that an element $h \in H$ is said to be a character element if there exists a character $\chi: G \rightarrow k^{\times}$such that for all $g \in G$,

$$
g^{-1} h g=\chi(g) h
$$

If $h$ is a nonzero character element, then the character $\chi$ is uniquely determined by the above equality, and $\chi=\chi^{h}$ is called a weight of $h$. A Hopf algebra $H$ is called a character if the group $G$ is abelian and $H$ is generated as an algebra with unity by character skew primitive elements. This is a large class of Hopf algebras containing, among others, quantum planes, Drinfeld-Jimbo quantized enveloping algebras $U_{q}(\mathfrak{g})$, and $G$-universal enveloping algebras of Lie color algebras.

If $R$ is an associative algebra acted on by a character Hopf algebra $H$, then any character skew primitive element $h \in L_{g}$ acts on $R$ as a $\chi^{h}(g)$-skew $g$-derivation. In this situation, any left module $M$ over the smash product $R \# H$ is a module over the skew polynomial ring $R[x ; g, h]$, where the action of $x$ coincides with the action of $h$, i.e., $x . m=h m$. Therefore, we are in a position to apply Propositions 7 , 9 and Corollary 8 to actions of character Hopf algebras.

Theorem 13. Let $H$ be a character Hopf algebra over the field $k$ of characteristic 0 and suppose that $\chi^{h}(g)$ is not an $n^{\text {th }}$ primitive root of unity $(n>1)$ for any character skew primitive element $h \in L_{g}$ and $g \in G$. Let $R$ be an associative $H$-module algebra. Then:

(1) Every finite dimensional irreducible left $R \# H$-module is completely reducible as a left $R$-module. In particular, $\mathcal{J}_{\text {fin }}(R) \subseteq \mathcal{J}_{\text {fin }}(R \# H)$.

(2) If $R$ is left socular, then irreducible left $R \# H$-modules are completely reducible as left $R$-modules. Thus $\mathcal{J}(R) \subseteq \mathcal{J}(R \# H)$.

\section{REFERENCES}

[1] Carl Faith, Algebra. II. Ring theory, Grundlehren der Mathematischen Wissenschaften, No. 191, Springer-Verlag, Berlin, 1976. MR0427349 (55 \#383)

[2] Edward Formanek and Arun Vinayak Jategaonkar, Subrings of Noetherian rings, Proc. Amer. Math. Soc. 46 (1974), 181-186. MR0414625(54 \#2725)

[3] K. R. Goodearl and E. S. Letzter, Prime ideals in skew and q-skew polynomial rings, Mem. Amer. Math. Soc. 109 (1994), no. 521, vi+106. MR.1197519 (94j:16051)

[4] P. Grzeszczuk, On G-systems and G-graded rings, Proc. Amer. Math. Soc. 95 (1985), no. 3, 348-352, DOI 10.2307/2045800. MR806068 (87a:16003)

[5] V. K. Kharchenko, An algebra of relatively primitive elements, Algebra i Logika 37 (1998), no. 2, 181-223, 249, DOI 10.1007/BF02671596 (Russian, with Russian summary); English transl., Algebra and Logic 37 (1998), no. 2, 101-126. MR.1672889 (99m:16061)

[6] V. Linchenko, S. Montgomery, and L. W. Small, Stable Jacobson radicals and semiprime smash products, Bull. London Math. Soc. 37 (2005), no. 6, 860-872, DOI 10.1112/S0024609305004662. MR2186719 (2006k:16084)

[7] Maria E. Lorenz and Martin Lorenz, Observations on crossed products and invariants of Hopf algebras, Arch. Math. (Basel) 63 (1994), no. 2, 119-127, DOI 10.1007/BF01189884. MR.1289292 (95f:16036)

[8] Martin Lorenz and D. S. Passman, Observations on crossed products and fixed rings, Comm. Algebra 8 (1980), no. 8, 743-779, DOI 10.1080/00927878008822488. MR566420(81c:16013)

Faculty of Computer Science, Biąystok University of Technology, Wiejska 45A, 15-351 Biąystok, Poland

E-mail address: piotrgr@pb.edu.pl 